\title{
NONLINEAR CONTROL, DISTURBANCE DECOUPLING AND LOAD ESTIMATION IN HVAC SYSTEMS
}

\author{
E. Semsar ${ }^{* *}$, M.J. Yazdanpanah ${ }^{*}$, C. Lucas ${ }^{*}$ \\ "Dept. of ECE, Faculty of Engineering, University of Tehran, Tehran, IRAN \\ *** Dept. of ECE, Faculty of Engineering, Concordia University, Montreal, Canada \\ Emails:_esemsar@ece.concordia.ca,yazdan@ut.ac.ir,.lucas@ipm.ir
}

\begin{abstract}
A new control methodology is introduced for nonlinear, MIMO Heating, Ventilating and Air Conditioning (HVAC) Systems. A feedback of states and disturbances is used for disturbance decoupling and model linearization purposes. The Back-stepping controller is applied to the system's linearized model, then. It is shown that in this way, heat and moisture loads can be compensated completely if considered as measurable disturbances. For non-measurable disturbances a stable observer is introduced to estimate them. The system has good and fast tracking, offset-free and smooth response with high disturbance decoupling and optimal energy consuming properties in the presence of a wide range of time-varying loads. Copyright (C) 2005 IFAC
\end{abstract}

Keywords: Feedback Linearization, Decoupling, Observer, nonlinear systems, tracking.

\section{NOMENCLATURE}

$h_{w}, h_{f g}$ Enthalpy of liquid water and water vapor

$W_{o}, W_{s}$ Humidity ratio of outdoor \& supply air

$W_{3} \quad$ Humidity ratio of thermal space

$V_{h e}, V_{s}$ Volume of heat exchanger \& thermal space

$M_{o}, Q_{o}$ Moisture \& heat loads

$T_{2}, T_{3} \quad$ Temperature of supply air \& thermal space

$f \quad$ Volumetric flow rate of recirculated air

$\rho, C_{p} \quad$ Air mass density, Specific heat of air

$T_{o} \quad$ Temperature of outdoor air

gpm Flow rate of chilled water

\section{INTRODUCTION}

HVAC systems are one of the most challenging plants in process control. The energy consumed by HVAC equipments constitutes $50 \%$ of the world energy consumption (Serrano and Reyes, 1999). In some cases the problem of HVAC control is inevitable for two main reasons. The first is to receive at an optimal consumption of energy. The second is that, in cases such as a metro system or in the towers with hundred stages, it is necessary to have a complete air conditioning system, which could keep the moisture, temperature, or pressure within acceptable ranges. There are some works done to achieve one or all of these purposes. In (Geng and Geary, 1993; Hartman, 1993) PID control and Direct Digital Control are used for temperature control. Intelligent methods have been used in (Ahmed, et al., 1996; Jian and Wenjian, 2000) for control and identification purposes. Zaheeruddin (1992, 1993) introduced an optimal controller for this system. In (Underwood, 2000) a robust methodology is applied to the system. For the purposes of control and minimization of actuator repositioning by Internal Model Control, (Omidi and Yazdanpanah, 2000) can be referred. In (Tigrek, et al., 2002) a nonlinear optimal controller was applied to this system, too.

Although many strategies have been used to improve the performance of these systems, there are many unsolved problems yet. The problem of load compensation can be considered as an example. It is important to have an acceptable air condition in the presence of time-varying disturbances such as heat and moisture loads with least energy consumption. This paper introduces a new approach using feedback linearization and Backstepping techniques for tracking control and disturbance decoupling goals. In the case of non-measurable disturbances, a stable observer is designed for estimation. The method is then applied to a nonlinear, MIMO, HVAC system. Simulation results show the advantages of the 
method in high disturbance decoupling, good tracking properties in the presence of time-varying loads and oscillation-free response. This in turn, results in longer life of actuators which is important from economical and industrial viewpoints.

The system model is first introduced in section 2 . Sections 3, 4 and 5 discuss the feedback linearization, observer design and Backstepping algorithms respectively. Finally simulations and conclusions are given in Sections 6 and 7.

\section{HVAC MODEL}

A single-zone Variable Air Volume (VAV), HVAC system is considered here. This system is a timedelayed, MIMO system in which one of the I/O channels has a right half plane zero and so is a nonminimum-phase system. Several models have been considered for HVAC systems in different references. In (Omidi and Yazdanpanh, 2000) a linear model of the system with a time delay and in (Underwood, 1999) a nonlinear SISO model is used. In some other works a bilinear model is considered for presenting the temperature(s) (Tigrek, et al., 2002), humidity or both of them (Serrano and Reyes, 1999). Here, the last one is considered, which presents both factors of temperature and humidity. In spite of (Serrano and Reyes, 1999), in which the thermal space temperature and humidity are the outputs, the outputs here are defined as thermal space and supply air temperatures while the humidity would be kept within an acceptable range. Moreover, to be more practical, the actuators' dynamics are considered as well. The mathematical model of the system without actuators' dynamics, is as in (1) and (2), where $x$ is the state vector, $z, y$ are the input and output vectors and $\omega$ is the disturbance vector of heat and humidity loads (Serrano and Reyes, 1999):

$$
\begin{gathered}
\dot{x}=f_{1} x z_{1}+g_{1} z+p_{1} \omega, y_{1}=x_{1}, y_{2}=x_{3} \\
f_{1}=\left[\begin{array}{ccc}
-\alpha_{1} 60 & \alpha_{2} 60 & \alpha_{1} 60 \\
0 & -\alpha_{1} 60 & 0 \\
\beta_{1} 45 & -\beta_{3} 45 & -\beta_{1} 60
\end{array}\right], p_{1}=\left[\begin{array}{cc}
\alpha_{3} & -\alpha_{3} h_{f g} \\
0 & \alpha_{4} \\
0 & 0
\end{array}\right] \\
g_{1}=\left[\begin{array}{cc}
-\alpha_{2} 60 W_{s} & 0 \\
\alpha_{1} 60 W_{s} & 0 \\
-\beta_{3} 60\left(.25 W_{o}-W_{s}\right)+\beta_{1} 15 T_{o} & 6000 \beta_{2}
\end{array}\right]
\end{gathered}
$$

Parameters and the numerical values are defined as follows (see nomenclature for definitions):

$$
\begin{gathered}
z_{1}=f, z_{2}=g p m, x_{1}=T_{3}, x_{2}=W_{3}, x_{3}=T_{2}, \omega_{1}=Q_{0} \\
\omega_{2}=M_{0}, \beta_{1}=1 / V_{h e}, \beta_{2}=1 / \rho C_{p} V_{h e}, \beta_{3}=h_{w} / C_{p} V_{h e} \\
\alpha_{1}=1 / V_{s}, \alpha_{2}=h_{f g} / C_{p} V_{s}, \alpha_{3}=1 / \rho C_{p} V_{s}, \alpha_{4}=1 / \rho V_{s}, \\
T_{o}=85^{\circ} \mathrm{F}, \rho=.074 \mathrm{lb} / \mathrm{ft}^{3}, V_{h e}=60 \mathrm{ft}^{3}, W_{o}=.0018, \\
V_{s}=58464 \mathrm{ft}^{3} C_{p}=.24 \mathrm{Btu} / \mathrm{lb} .{ }^{\circ} \mathrm{F}, W_{s}=.007
\end{gathered}
$$

It is obvious that if both input and disturbance vectors are zero, $\dot{x}$ would be zero, which has a physical meaning, as when flow rate of water and air, moisture and heat loads are zero, then the temperature and humidity of thermal space would remain invariant and so their derivative is zero. Here the control signal is implemented to liquid valve with the dynamic model as (Underwood, 1999):

$$
G(s)=z(s) / u(s)=k /\left(1+\tau_{v} s\right)
$$

with $\tau_{v}$ : valve time constant, $u(s)$ and $z(s)$ : control signals applied to actuator and plant respectively, $k$ : a constant. Parameter values are $\tau_{v}=.008 \mathrm{hr}, k=5$

The augmented model of the system and actuators will be as in (4) in which, $X=\left[\begin{array}{ll}x & z\end{array}\right]^{T}$ :

$$
\begin{aligned}
& \dot{X}=F(X)+G(X) u+P(X) \omega, y=\left[\begin{array}{ll}
x_{1} & x_{3}
\end{array}\right]^{T} \\
& G(X)=\left[\begin{array}{ccccc}
0 & 0 & 0 & k / \tau_{v} & 0 \\
0 & 0 & 0 & 0 & k / \tau_{v}
\end{array}\right]^{T}, F(X)= \\
& {\left[\begin{array}{lllll}
\gamma_{1} z_{1} & \gamma_{2} z_{1} & \gamma_{3} z_{1}+\gamma_{4} z_{2} & -z_{1} / \tau_{v} & -z_{2} / \tau_{v}
\end{array}\right]^{T} \text {, }} \\
& P(X)=\left[\begin{array}{ccccc}
\alpha_{3} & 0 & 0 & 0 & 0 \\
-\alpha_{3} h_{f g} & \alpha_{4} & 0 & 0 & 0
\end{array}\right]^{T} \\
& \gamma_{1}=\alpha_{1} 60\left(x_{3}-x_{1}\right)-\alpha_{2} 60\left(W_{s}-x_{2}\right) \\
& \gamma_{2}=\alpha_{1} 60\left(W_{s}-x_{2}\right), \gamma_{4}=-6000 \beta_{2} \\
& \gamma_{3}=\beta_{1} 15\left(3 x_{1}+T_{o}-4 x_{3}\right) \\
& -\beta_{3} 60\left(.25 W_{o}+.75 x_{2}-W_{s}\right)
\end{aligned}
$$

\section{LINEARIZATION VIA FEEDBACK}

\subsection{Relative degree}

Relative degree is the number of times, the output should be differentiated to have the input be appeared and the relative degree for a MIMO system is defined in (Isidori, 1995) as follows. Consider the system as:

$$
\left\{\begin{array}{l}
\dot{X}=F(X)+\sum_{i=1}^{m} g_{i}(X) u_{i}+P(X) \omega= \\
F(X)+G(X) u+P(X) \omega, X \in R^{n}, u_{i}, \omega \in R \\
y_{i}=h_{i}(X) \quad i=1, \ldots, m, \quad y_{i} \in R
\end{array}\right.
$$

It has a vector input relative degree around $X_{0}$ as $r=\left\{r_{1}, \ldots, r_{m}\right\}$, if the following conditions are met:

$$
\text { i) } L_{g_{j}} L_{F}^{k} h_{i}=0,1 \leq j \leq m, 1 \leq i \leq m, k<r_{i}-1 \text { (6-a) }
$$

$L_{F}^{k} h$, Lie derivative, is the $\mathrm{k}^{\text {th }}$ derivative of $h(X)$ with respect to $X$ along $F$ (Isidori, 1995).

ii) $A(X)$ is nonsingular in a neighbourhood of $X_{0}$

$$
A(X)=\left[\begin{array}{ccc}
L_{g_{1}} L_{F}^{\eta^{1}-1} h_{1}(X) & \cdots & L_{g_{m}} L_{F}^{r_{1}-1} h_{1}(X) \\
\vdots & \ddots & \vdots \\
L_{g_{1}} L_{F}^{r_{m}-1} h_{m}(X) & \cdots & L_{g_{m}} L_{F}^{r_{m}^{-1}} h_{m}(X)
\end{array}\right](6-\mathrm{b})
$$

\subsection{Relative degree in HVAC system}

Considering the model in (4), after some calculations, it can be shown that for an HVAC system:

$$
A(X)=\left[\begin{array}{cc}
\gamma_{1} k_{1} / \tau_{1} & 0 \\
\gamma_{3} k_{1} / \tau_{1} & \gamma_{4} k_{2} / \tau_{2}
\end{array}\right], r=\{2,2\}(7)
$$




\subsection{Exact linearization via feedback: MIMO case}

As mentioned in (Isidori, 1995), feedback linearization is applicable to MIMO systems with disturbances in their model, as HVAC model discussed here. It is implied there that there exist a feedback for the system described in (5), which transforms it to the normal form. This feedback can render the outputs independent of the disturbances. The important point is that the disturbances have to be available, or observable to be estimated:

Lemma 1: 1. There exists a feedback of the form $u=\alpha(X)+\beta(X) v$, which renders the output of (5) independent of the disturbance $\omega$ if and only if $L_{P} L_{F}^{k} h_{i}(X)=0,0 \leq k \leq r_{i}-1,1 \leq i \leq m$

this means that relative degree with respect to inputs should be less than the disturbances' relative degree, 2. There exists a feedback of the form $u=\alpha(X)+\beta(X) v+\gamma(X) \omega$ which renders the output of (5) independent of the disturbance $\omega$ if and only if $L_{P} L_{F}^{k} h_{i}(X)=0,0 \leq k \leq r_{i}-2,1 \leq i \leq m$

Proof: By differentiating the output relation and using the definition of input relative degree.

There are so many systems, which cannot guarantee the conditions mentioned in lemma 1 . Here an special conditions, more than lemma 1 , are mentioned as a new introduced lemma in which linearization and disturbance decoupling would be possible.

Lemma 2: There exists a feedback of the form $u=\alpha(X)+\beta(X) v+\gamma(X) \omega$, which renders the output of (5) independent of the disturbance $\omega$ if i) $L_{P} L_{F}^{k} h_{i}(X)=0$ for $0 \leq k<r_{i}-2,1 \leq i \leq m$ (10-a)

ii) $L_{P} L_{F}^{r_{i}-2} h_{i}(X)$ is independent of state variables.

Introducing a new coordinate transformation, different from the one in (Isidori, 1995), this lemma can be proved. Here a brief proof is brought.

Proof: By differentiating the output relation continuously it will be seen that:

$$
\begin{aligned}
& \dot{y}_{i}=L_{F} h_{i}(X) \\
& \vdots \\
& y_{i}^{\left[r_{i}-1\right]}=L_{F}^{r_{i}-1} h_{i}(X)+\left(L_{P} L_{F}^{r_{i}-2} h_{i}(X)\right) \omega \\
& y_{i}^{\left[r_{i}\right]}=L_{F}^{r_{i}} h_{i}(X)+\left(L_{g} L_{F}^{r_{i}-1} h_{i}(X)\right) u+ \\
& \left(L_{P} L_{F}^{r_{i}-1} h_{i}(X)\right) \omega=v_{i}
\end{aligned}
$$

By introducing new coordinate as:

$$
\begin{gathered}
\xi_{1}^{i}=h_{i}(X), \xi_{2}^{i}=L_{F} h_{i}(X), \ldots, \xi_{r_{i}-1}^{i}=L_{F}^{r_{i}-2} h_{i}(X) \\
\xi_{r_{i}}^{i}=L_{F}^{r_{i}-1} h_{i}(X)+\left(L_{P} L_{F}^{r_{i}-2} h_{i}(X)\right) \omega
\end{gathered}
$$

and using ii) in lemma 2 :

$$
\frac{\partial}{\partial X}\left(L_{P} L_{F}^{r_{i}-2} h_{i}(X)\right)=0, \quad i=1, \ldots, m
$$

It can be shown that by using the properties of Lie derivative and the definition of input relative degree, the following equations are held:

$$
\begin{aligned}
& \dot{\xi}_{1}^{i}=\xi_{2}^{i}, \ldots, \quad \dot{\xi}_{r_{i}-1}^{i}=\xi_{r_{i}}^{i} \\
\dot{\xi}_{r_{i}}^{i}= & L_{F}^{r_{i}} h_{i}(X)+\left(L_{g} L_{F}^{r_{i}-1} h_{i}(X)\right) u+ \\
& \left(L_{P} L_{F}^{r_{i}-1} h_{i}(X)\right) \omega=v_{i} \\
y_{i}= & \xi_{1}^{i}
\end{aligned}
$$

by using $\dot{\xi}_{1}^{i}$ definition, control law can be written as:

$$
\begin{gathered}
u=\alpha(X)+\beta(X) v+\gamma(X) \omega \\
\alpha(X)=-A^{-1} b, \beta(X)=A^{-1}, \gamma(X)=-A^{-1} c \\
c(X)=\left[\begin{array}{c}
L_{P} L_{F}^{r_{1}-1} h_{1}(X) \\
\vdots \\
L_{P} L_{F}^{r_{m}-1} h_{m}(X)
\end{array}\right], b(X)=\left[\begin{array}{c}
L_{F}^{r_{1}} h_{1}(X) \\
\vdots \\
L_{F}^{r_{m}} h_{m}(X)
\end{array}\right]
\end{gathered}
$$

It is clear that applying a nonlinear feedback of the states and disturbances as in (14) can linearize the system model. Hence the output is obtained independent of the disturbances, hence decoupled from the disturbances, and the proof is complete.

It is worthy to mention that although the transformed states $\xi^{i}$ are functions of the disturbance $\omega$, this does not change the problem of achieving disturbance decoupling. The reason is that the system can be changed into the normal form without considering the definition of $\xi^{i}$. It means that even if $\xi^{i}$ has a term of $\omega$ in its definition, the effect of it can be considered together with the effect of $\omega$ appeared in the feedback in the form of $u=\alpha(X)+\beta(X) v+\gamma(X) \omega$. In other words this feedback depends on $\omega$ and hence, defining its coefficients as functions of $\omega$ does not result in a problem in general cases.

\subsection{Linearization via feedback in HVAC system}

In the case of HVAC system these relations are held: $L_{P} L_{F}^{k} h_{1}(X) \neq 0$ For $(0 \leq k \leq 2-2=0 \Rightarrow k=0)$ $L_{P} L_{F}^{k} h_{2}(X)=0$ For $(0 \leq k \leq 2-2=0 \Rightarrow k=0)$

So no parts of lemma 1 can be applied to it. Now try lemma 2. Condition (10-a) is achieved automatically, as no value can be found for $k$. considering (10-b):

$$
\begin{aligned}
& L_{P} L_{F}^{r_{i}-2} h_{i}(X)=L_{P} L_{F}^{0} h_{i}(X)=L_{P} h_{i}(X)= \\
& {\left[\begin{array}{lll}
\alpha_{3} & -\alpha_{3} h_{f g}
\end{array}\right]^{T} \text { for } i=1 \quad \& \quad\left[\begin{array}{ll}
0 & 0
\end{array}\right]^{T} \text { for } i=2}
\end{aligned}
$$

which are independent of state variables. So this lemma is applicable to the HVAC system. Now a feedback as in (14) with a coordinate transformation in the form of (12) can transform the system into the linear form (13). The transformation is as:

$$
\begin{aligned}
& \xi_{1}^{1}=x_{1}, \quad \xi_{2}^{1}=\gamma_{1} z_{1}+\alpha_{3}\left(\omega_{1}-h_{f g} \omega_{2}\right) \\
& \xi_{1}^{2}=x_{3}, \quad \xi_{2}^{2}=\gamma_{3} z_{1}+\gamma_{4} z
\end{aligned}
$$

So the control law, $u$, in (14) with $A(X)$ as in (7),

$$
c(X)=\left[\begin{array}{cc}
-\alpha_{3} \alpha_{1} z_{1} 60 & 60 z_{1}\left(-\alpha_{3} \alpha_{1} h_{f g}+\alpha_{2} \alpha_{4}\right) \\
\alpha_{3} \beta_{1} z_{1} 45 & 45 z_{1}\left(-\alpha_{3} \beta_{1} h_{f g}-\beta_{3} \alpha_{4}\right)
\end{array}\right]
$$


and $b(X)=\left[\begin{array}{ll}L_{F}^{2} h_{1}(X) & L_{F}^{2} h_{2}(X)\end{array}\right]^{T}$ will transform the nonlinear system (4) into the linear form of (13). So there will be a linear model with the outputs, decoupled from the disturbances and a suitable controller based on control goals can be designed for it. Here Backstepping is used for this purpose.

\section{LOAD ESTIMATION}

As it was said in the previous section, a nonlinear system in the form of (5), which satisfies special conditions, can be transformed into the linear form of (13) by a nonlinear feedback described in (14). However, in order to use (14), it is necessary to know disturbance vector or to estimate it somehow. In some cases the disturbances are available but here, it is assumed that they are not measurable but have constant values, in comparison with the system time constant, which results in the following equation:

$$
\dot{\omega}=0
$$

In this way the equation (14) would be replaced with

$$
u=\alpha(X)+\beta(X) v+\gamma(X) \hat{\omega}
$$

in which $\hat{\omega}$, the output of observer, is the estimated value for $\omega$. Here an observer is introduced which has the inputs and states of the original system as its inputs and the estimated disturbances as its outputs.

\subsection{Observer design}

It simply can be shown that the system described in (1), (2) is observable (Serrano and Reyes, 1999). Therefore an observer can be designed for it. Considering the system in (1), by introducing $\tilde{X}=\left[\begin{array}{ll}x^{T} & \omega^{T}\end{array}\right]^{T}$ and using (16), augmented system would be given by:

$$
\begin{aligned}
& \dot{\widetilde{X}}=\left[\begin{array}{cc}
0 & p_{1} \\
0 & 0
\end{array}\right]\left[\begin{array}{c}
x \\
\omega
\end{array}\right]+\left[\begin{array}{cc}
f_{1} & 0 \\
0 & 0
\end{array}\right]\left[\begin{array}{c}
x \\
\omega
\end{array}\right] z_{1}+\left[\begin{array}{c}
g_{1} \\
0
\end{array}\right] z \\
& =\bar{A} \tilde{X}+\bar{N} \widetilde{X} z_{1}+\bar{B} z
\end{aligned}
$$

Now by partitioning this system as $\bar{X}_{1}=x$, $\bar{X}_{2}=\omega$, the following relations can be written:

$\left\{\begin{array}{l}\dot{\bar{X}}_{1}=\bar{A}_{11} \bar{X}_{1}+\bar{A}_{12} \bar{X}_{2}+\bar{N}_{11} \bar{X}_{1} z_{1}+\bar{N}_{12} \bar{X}_{2} z_{1}+\bar{B}_{1} z \\ \dot{\bar{X}}_{2}=\bar{A}_{21} \bar{X}_{1}+\bar{A}_{22} \bar{X}_{2}+\bar{N}_{21} \bar{X}_{1} z_{1}+\bar{N}_{22} \bar{X}_{2} z_{1}+\bar{B}_{2} z\end{array}\right.$ in which $\bar{N}_{i j}, \bar{A}_{i j}, i, j=1,2$ are the corresponding partitions of $\bar{N}, \bar{A}$ according to $\bar{X}_{1}, \bar{X}_{2}$. After modifications the following equation is obtained, in which $\Gamma=\hat{\bar{X}}_{2}-\bar{L}_{\bar{X}}$ and $\hat{\bar{X}}_{2}$ is estimation for $\bar{X}_{2}$ :

$$
\begin{aligned}
\dot{\Gamma}= & {\left[\bar{A}_{22}+\bar{N}_{22} z_{1}-\bar{L}\left(\bar{A}_{12}+\bar{N}_{12} z_{1}\right)\right] \Gamma+\left(-\bar{L}_{11}+\bar{A}_{21}\right) \bar{X}_{1} } \\
& +\left(\bar{B}_{2}-\bar{L}_{1}\right) z+\left[\bar{A}_{22}+\bar{N}_{22} z_{1}-\bar{L}\left(\bar{A}_{12}+\bar{N}_{12} z_{1}\right)\right] \bar{L} \bar{X}_{1} \\
& +\left(\bar{N}_{21}-\bar{L}_{11}\right) \bar{X}_{1} z_{1}
\end{aligned}
$$

This equation shows the observer's dynamics to be added to the original system dynamics. As mentioned before $\bar{X}_{1}$ and $z$ are the available state and input vectors, $\bar{N}_{i j}, \bar{A}_{i j}, i, j=1,2$ are known and $\bar{L}$ is the observer gain matrix to be designed such that it guarantees the stability of the observer dynamics. It can be shown that considering $\bar{e}=\hat{\bar{X}}_{2}-\bar{X}_{2}$ the estimation error dynamic would be:

$$
\dot{\bar{e}}=\left[\bar{A}_{22}+\bar{N}_{22} z_{1}-\bar{L}\left(\bar{A}_{12}+\bar{N}_{12} z_{1}\right)\right] \bar{e}=\mu \bar{e}
$$

Now in order to make (20) asymptotically stable around the origin, $\mu$ should be negative definite. A value of $\bar{L}$, which meets this condition and provides a fast response for HVAC system is given by:

$$
\bar{L}=\left[\begin{array}{lll}
1 & 0 & 0 \\
0 & 1 & 0
\end{array}\right] \times 10^{7}
$$

Hence $\Gamma$ can be obtained from (19) by using (21) and $\hat{\bar{X}}_{2}$ is given by:

$$
\hat{\omega}=\hat{\bar{X}}_{2}=\Gamma+\bar{L} \bar{X}_{1}
$$

\section{BACKSTEPPING METHOD}

Consider the system in (23). By input transformation (24), it can be written in the form of (25):

$$
\begin{aligned}
& \left\{\begin{array}{c}
\dot{\eta}=f(\eta)+g(\eta) \zeta, \quad \eta \in R^{n}, \xi \in R \\
\dot{\zeta}=f_{a}(\eta, \zeta)+g_{a}(\eta, \zeta) u_{a}, \quad u_{a} \in R
\end{array}\right. \\
& u_{a}=\frac{1}{g_{a}(\eta, \zeta)}\left[u-f_{a}(\eta, \zeta)\right] \\
& \left\{\begin{array}{c}
\dot{\eta}=f(\eta)+g(\eta) \zeta \\
\dot{\zeta}=u
\end{array}\right.
\end{aligned}
$$

Choose $\zeta=\varphi(\eta)$ such that it makes the first equation in (25) asymptotically stable around the origin, $\eta=0, \zeta=0$. Khalil (1996) shows that using a lyapunov function as in (26), the control law in (27) makes the whole system (25) asymptotically stable:

$$
\begin{aligned}
& V_{a}(\eta, \zeta)=V(\eta)+1 / 2 z^{2}, z=\zeta-\varphi(\eta) \\
& \frac{\partial V}{\partial \eta}[f(\eta)+g(\eta) \varphi(\eta)] \leq-W(\eta), \quad W(\eta): P . D .(26-\mathrm{b}) \\
& u=\frac{\partial \varphi}{\partial \eta}[f(\eta)+g(\eta) \zeta]-\frac{\partial V}{\partial \eta} g(\eta)-k[\zeta-\varphi(\eta)](27)
\end{aligned}
$$

$k$ is a design parameter, shows the negativity of $\dot{V}$ and affects robustness, stability, and response speed. It can be shown that a system described in normal form as in (13) is a special case of the system in the form of (25) with these definitions:

$$
\begin{aligned}
& f\left(\eta^{i}\right)=\left[\begin{array}{cccc}
0 & 1 & 0 & \cdots \\
\vdots & \vdots & \vdots & \vdots \\
0 & & \cdots & 1 \\
0 & 0 & \cdots & 0
\end{array}\right] \eta^{i}, g\left(\eta^{i}\right)=\left[\begin{array}{c}
0 \\
0 \\
\vdots \\
1
\end{array}\right], u_{i}=v_{i}, \\
& \eta^{i}=\left[\xi_{1}^{i} \cdots \xi_{r_{i}-1}^{i}\right]^{T}, \zeta^{i}=\xi_{r_{i}}^{i}, z=\zeta^{i}-\varphi\left(\eta^{i}\right)(28)
\end{aligned}
$$

The candidates of $V, \varphi$ satisfying the conditions are:

$$
V=1 / 2\left\|\eta^{i}\right\|^{2}, \varphi\left(\eta^{i}\right)=\left[\begin{array}{lll}
(-1)^{r_{i}-1} & 0 & \ldots
\end{array}\right]_{1 \times\left(r_{i}-1\right)} \eta^{i}
$$

\subsection{Applying Backstepping to the HVAC system}

As it was mentioned previously, HVAC model used 
in this paper has a vector relative degree for the input as $r=\{2,2\}$. First we consider that the control goal is to regulate the output around a point $y_{d}^{i}$ not necessarily the origin. So first we shift the model to have the regulation at the origin of the new system:

$$
\left(y^{i} \rightarrow y_{d}^{i}\right) \Rightarrow\left(\xi_{1}^{i} \rightarrow \xi_{1 d}^{i} \Rightarrow \widetilde{\xi}_{1}^{i}=\xi_{1}^{i}-\xi_{1 d}^{i} \rightarrow 0\right)(30)
$$

The Backstepping controller for the system in (15) is: $r_{i}=2 \Rightarrow\left(\eta^{i}=\tilde{\xi}_{1}^{i}, \quad \zeta^{i}=\xi_{2}^{i}, \quad u_{i}=v_{i}\right) \Rightarrow$

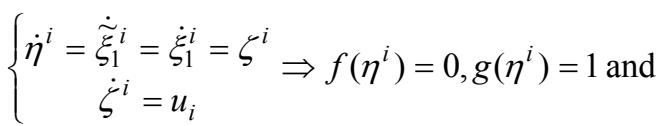
according to (29), $V=.5\left(\eta^{i}\right)^{T} \eta^{i}, \varphi\left(\eta^{i}\right)=-\eta^{i}$ and: $V_{a}=V+.5 z^{2}, z=\zeta^{i}+\eta^{i}$

hence by applying the coordinate shift as in (30), the control law for $u\left(\zeta^{i}(X)\right)$ is given by:

$$
u_{i}=v_{i}=-\left(1+k_{i}\right)\left(\xi_{1}^{i}+\xi_{2}^{i}-\xi_{1 d}^{i}\right), i=1,2
$$

the $k_{i}$ should be designed according to the design goals such as robustness, response speed, etc.

\subsection{Control algorithm}

Finally to conclude the control strategy we can present the following algorithm:

1. If disturbance vector is measurable use control law in (14) and transformation in (15) to transform the nonlinear system (4) to linear one (13). If disturbance vector is not available use its estimated value using observer dynamics in (19) with gain matrix in (21) and consider (17) as control law instead of (14).

2. Calculate $v_{i}$ according to (32) by substituting corresponding value for $\xi$ from (15).

3. Having $v_{i}, \omega(\hat{\omega})$ and $X$ control law $u$ can be obtained from (14) $((17))$.

The control $u$ is applied directly to the system in (4).

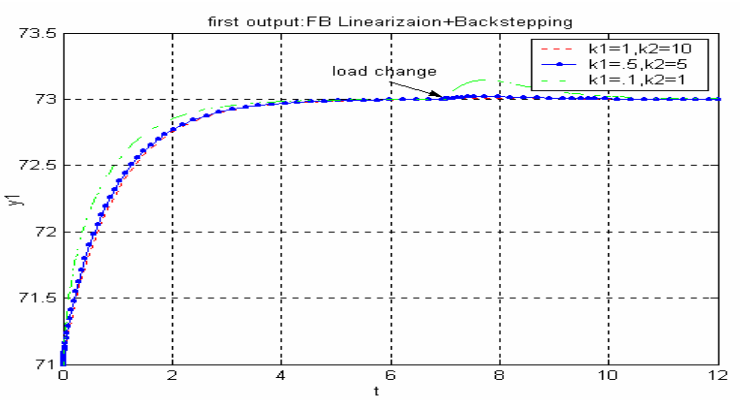

Fig. 1. $1^{\text {st }}$ output response for different values of $k$

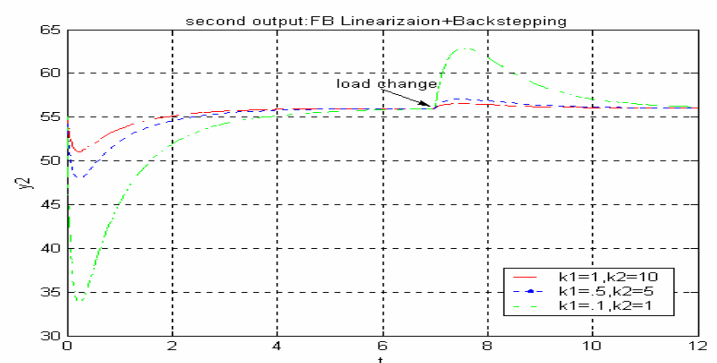

Fig. 2. $2^{\text {nd }}$ output response for different values of $k$

\section{SIMULATIONS}

Simulations presented here, are the results of applying proposed method to a nonlinear, MIMO, HVAC system. In figures 1, 2 first and second outputs and in figures 3, 4 first and second control signals are presented. The output set points are 73 and $56^{\circ} \mathrm{F}$ and initial state vector is $X_{0}=\left[\begin{array}{lll}71 & .0092 & 55\end{array}\right]^{T}$. There are load changes at $t=7 \mathrm{hr}$ from 289897 to 360000 and 166 to 197 for both disturbances ( $25 \%$ ). It is seen that responses are adapted to load changes very soon with no offsets remained in them, which show the good capabilities of the proposed controller in overcoming disturbances and exact regulation properties. These can be compared with the results in (Serrano and Reyes, 1999) for almost less percentage of the load changes $(15 \%)$. In their work, both output responses have offset $\left(3^{\circ} \mathrm{F}\right)$ after load variations. It was mentioned previously that gain $k$ in (27) shows the negativity of $\dot{V}$. In all figures, there are comparisons between the outputs for different values of this parameter and as it become larger, robustness and speed of responses, improve. Moreover, the value of overshoots and undershoots in output decreases significantly. The only disadvantage is the sharpness of the control signal when load changes in figures 3, 4. This is not a problem since the minimum time constant of actuators, $.008 h r=29 s$ is met. Also, the value of the second state, humidity percentage, is kept within an acceptable range of $(.008, .013)$ as in figure 5. Figures 6, 7 and 8 compare the responses of proposed controller and a PID designed by ZieglerNichols method. In figures 6 and 7 first and second outputs are compared. It is seen that proposed controller, F.L.+Bs, has no oscillations in comparison with PID, while it has almost the same speed. As PID does not have enough robustness to tolerate timevarying loads, its simulations are done in presence of constant loads. In figure 8, second control signals for two controllers are compared. It is seen that proposed method has an oscillation-free signal which means less actuator repositioning and latter, in turn, results in longer life of actuators. This is an important advantage of this method from economical and industrial aspects, which results in an optimal energy consumption and can be measured via the index:

$$
E=\int_{0}^{t}\left(u-u_{e q}\right)^{2} d t
$$

$u_{e q}$ is steady state value of control signal. This index measures both value and number of control signal oscillations. The values of $E$ are 1293 and 97 for PID and proposed method respectively. There is a decrease of $90 \%$ using the introduced method comparing to PID. Results of comparing proposed approach with other techniques of adjusting PID follow the same tendency, which shows proposed technique superiority to PID for HVAC control. 


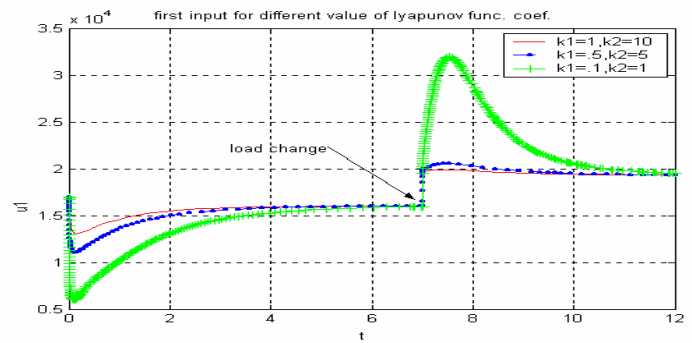

Fig. 3. $1^{\text {st }}$ control signal for different values of $k 7$.

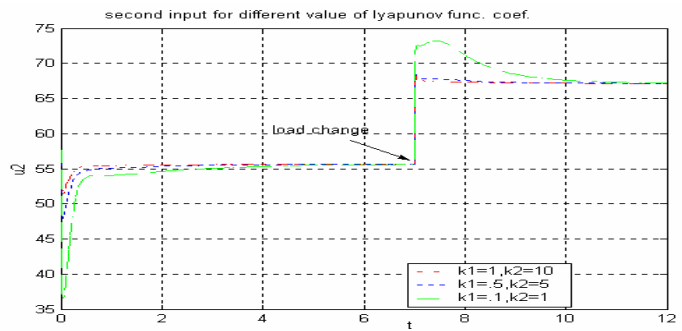

Fig. 4. $2^{\text {nd }}$ control signal for different values of $k$

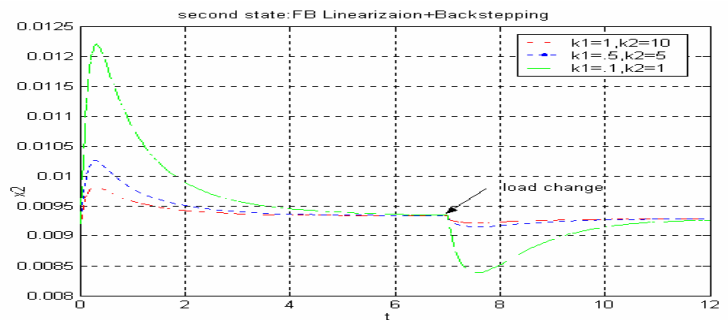

Fig. 5. $2^{\text {nd }}$ state response for different values of $k$

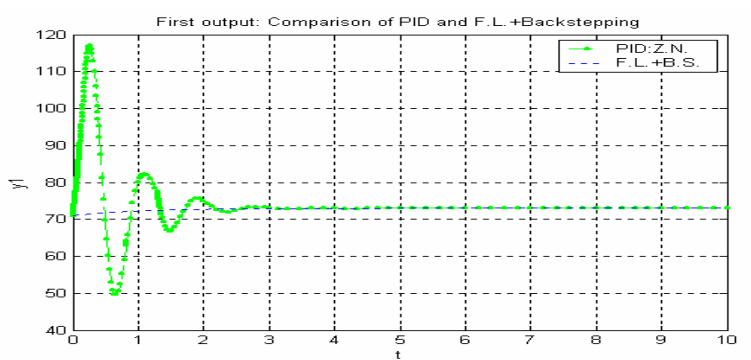

Fig. 6. $1^{\text {st }}$ output for PID and F.L.+Bs controllers

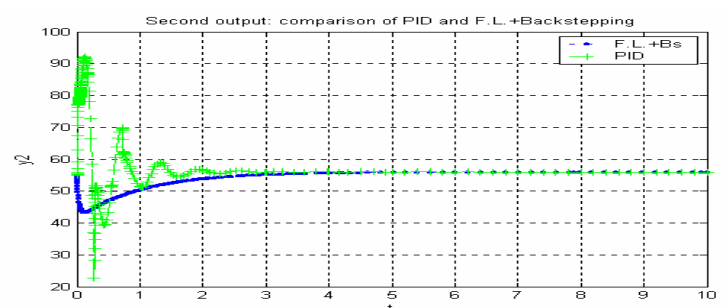

Fig. 7. $2^{\text {nd }}$ output for PID and F.L.+Bs controllers

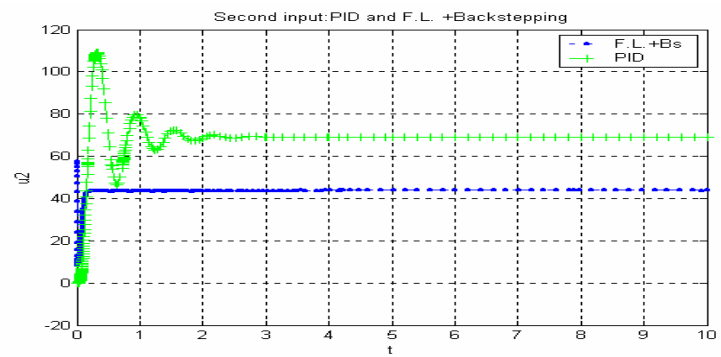

Fig. 8. $2^{\text {nd }}$ control signal for PID and F.L. + Bs

\section{CONCLUSIONS}

An algorithm was introduced for disturbance decoupling and tracking in nonlinear MIMO systems. In this method first the nonlinear model is transformed into a linear one using a nonlinear feedback of states and disturbances. Wherever the disturbances are not available, a stable observer is designed to estimate them. Finally a state feedback is designed by Backstepping for the system in normal form and control law is applied to original system. Applying the method to a MIMO, nonlinear, HVAC system shows the effectiveness of algorithm in reaching the pre-specified design goals such as output regulation, disturbance decoupling and offset removal in the presence of wide- range time-varying loads. Also, the responses were oscillation-free compared with other common control strategies. This means less actuator repositioning which in turn, results in longer life of actuators. Hence this method is more beneficial from an industrial viewpoint.

\section{REFERENCES}

Ahmed, O., J. Mitchell and S. Klein (1996). Application of GRNN in HVAC Process Identification and Control. ASHRAE, 102.

Geng, G. and G. Geary (1993). On Performance and Tuning of PID Controllers in HVAC Systems. IEEE CCA, 2, pp. 819-824.

Hartman, T.B. (1993). Direct Digital Controls for HVAC Systems, Mc Graw-Hill.

Isidori, A. (1995). Nonlinear Control Systems, Springer-Verlag.

Jian, W. and C. Wenjian (2000). Development of an Adaptive Neuro-Fuzzy Method for Supply Air Pressure Control in HVAC System. IEEE conf. Sys., Man, and Cybernetics, 5 , pp. 3806 -3809.

Khalil, H.K. (1996) Nonlinear Systems, Prentice Hall.

Omidi, M.R. and M.J. Yazdanpanah (2000). Minimization of Actuator Repositioning Using Internal Model Control. Proc. Fourth Portuguese, Automatic Control Conf.

Serrano, B.A. and M.V. Reyes (1999). Nonlinear Control of a Heating, Ventilating and Air Conditioning System with Thermal Load Estimation. IEEE Trans. Con. Sys. Tech., 7, pp. 5663.

Tigrek, T., S. Dasgupta and T.F. Smith (2002). Nonlinear Optimal Control of HVAC Systems. Proc. IFAC World Congress.

Underwood, C.P. (1999). HVAC Control systems: Modeling, Analysis and design. E \& FN SPON.

Underwood, C.P. (2000). Robust Control of HVAC Plant II: Controller Design. CIBSE, 21, No. 1

Zaheeruddin, M. (1992). Optimal Control of a Single Zone Environmental Space. Building and Environment journal.

Zaheeruddin, M. (1993). The Design and Simulation of a Sub-optimal Controller for space Heating. ASHRAE Trans 